\title{
Research on Integrated Cooperative Design of Building Structure Based on Building Information Model
}

\author{
Rulun Peng ${ }^{1}, \quad$ Tianyi Liu ${ }^{1}, \quad$ Min Zhang $^{1}$ \\ ${ }^{1}$ CHONGQING COLLEGE OF ARCHITECTURE AND TECHNOLOGY, CHONG QING， 401331
}

\begin{abstract}
With the rapid development of the socialist market economy, the development of the construction industry and the information industry has become more mature and sound. However, the practical application of information technology in the existing construction industry still faces corresponding problems, lacking sufficient completeness and specificity. This article will specifically discuss and analyze the building structure integrated collaborative design program of building information model, in order to provide a foundation for the development of modern construction industry.
\end{abstract}

\section{Introduction}

With the rapid development of modern electronic information technology, it has already occupied a very important position in China's actual construction and development. In the current development of China's construction industry, engineering design mainly uses twodimensional design as the core support, and in actual construction, information is mainly disseminated in various forms. The final delivery of architectural design is usually completed by means of two-dimensional design. Although the two-dimensional design has been applied for a long time, it still faces the limitations of many influencing factors when the actual design plan is modified. This will eventually lead to a lot of human resource costs. The emergence of building model technology can greatly reduce the development difficulties faced by the construction industry. But in fact, due to the lack of application standards and design patterns of building information models, this has led to various problems in actual construction. This article believes that it is extremely important to discuss the integrated collaborative design of building structure of building information model.

\section{Overview of the Development of Building Information Modeling Technology}

\subsection{Building Information Model}

The building information model aims to realize the effective integration of construction projects and information technology, and it plays a very important role in the construction and development of the modern construction industry. At this stage, some people still think that building information models are three-dimensional modeling tools. But in fact, it is far from the case. The role played by the thinking model included in the building information model cannot be ignored. It can gather various factors in the implementation of specific construction projects. In this way, accurate and specific calculation processing can be completed to ensure the stable progress of various standard activities in specific construction activities [1]. Its core meaning is to greatly promote the precise narrative expression of design information related to construction projects. Its main features include the following points.

\subsubsection{Information Linkage and Integration}

In the process of creating models, building information technology uses three-dimensional digital model technology and related parameters to create models, which are essentially different from traditional design solutions. The traditional scheme mainly takes the drawing of points, lines, and surfaces as the core support to complete the construction of the model, and determine the basic form of the building through a large number of combinations. And building information technology is to construct and process the doors, windows, and beam structures involved in the building. It can set the parameters through the corresponding building relationship, and finally complete the modification processing of the building relevance. In the actual implementation process, the role played by the building information model cannot be ignored, and the information data included in it is even more numerous. There are also essential differences in the information storage modes used by different forms of construction software, which can basically be divided into two types: basic data and additional data [2]. Building information model mainly includes data model and behavior model. The above two models enable the actual meaning of data to be effectively presented. This can provide a pavement for the normal development of subsequent construction 
activities.

\subsubsection{Information Coordination and Consistency}

The core of traditional architectural model design is twodimensional design mode, and the core of building information model is three-dimensional model system. But in the specific operation, the relevant workers can combine their actual needs to realize the conversion from threedimensional to two-dimensional, which can make it clear that they have the corresponding consistency characteristics. At the same time, during specific operations, building information technology has gradually developed into a key factor in the information assurance link. It can promote the satisfaction of information sharing processing and other characteristics in the process of actual model construction, and reduce the probability of error in actual drawing. Meanwhile, it can also achieve synchronization update optimization with drawings. What is reflected here is the coordination of the building information model, which can ultimately effectively improve the overall efficiency and quality of the design process.

\subsubsection{Information Collaborative Design}

As a brand-new architectural design platform, building information model can provide a more solid communication bridge for architectural design units, engineering construction units and project owners, and is used to provide multiple information content for project processing to multiple parties. Through the effective application of the collision detection function in the building information model system, it can combine the design principles followed by different professions to realize effective supervision and control of different component objects. This can ensure that designers can find and solve problems in existing designs in time. In this case, the communication efficiency and communication quality of professionals will be significantly improved. In the meantime, the time invested in construction design will also be effectively reduced, and the quality of the current building system can be fully guaranteed [3]. For example, the IFC standard is an important standard concept in the design of modern building information models. From the initial development to the present, the IFC standard system has become more mature and perfect. After a long period of development, the standard has become a principle that the Chinese construction industry needs to fully comply with. The development process is shown in Figure 1.

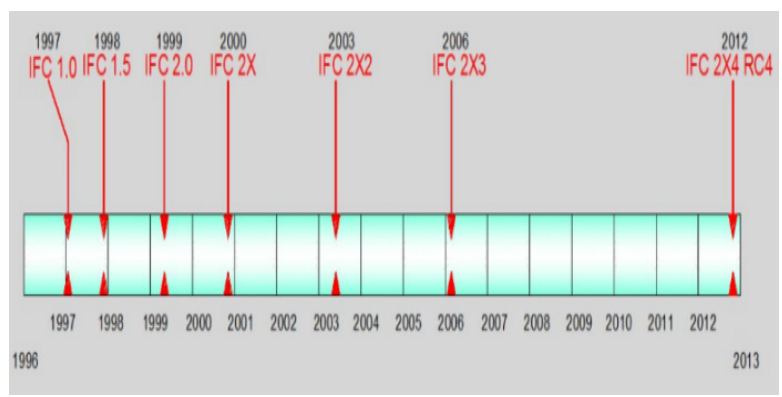

Fig1. IFC Standard Development History

\subsection{Application of Building Information Model}

Judging from the current situation, there are still certain problems in the use of building information models. The first is the problem of the staff, who are accustomed to the application of two-dimensional design concepts, and cannot successfully master the technology of operating building information modeling in a short time, and cannot design a more complete three-dimensional design method. For this reason, relevant leaders need to have a forwardlooking development vision and regularly organize employees to participate in the training of new technologies [4]. The second is the technical level. Many contracting companies cannot master more advanced hardware and software application technologies. Although there are many software operating systems on the market, many of the software's designs are not perfect and cannot meet the actual needs of today's society. They still need to rely on manual operations when drawing pictures. This leads to a low overall work efficiency. Not only that, many information models require higher computer hardware when they are constructed. These issues require relevant technical personnel to think in many ways to enhance the use value of the system. Only in this way can the subsequent system use problems be improved. Finally, there are many problems in the management process. For example, three-dimensional drawing technology does not have high efficiency when implemented.

\subsection{Technical Standards for Building Information Modeling}

In the specific construction activities, the building information model should be set with matching technical standards constraints. Only by fully complying with specific technical standards can it accurately demonstrate the role of building information models, and can be effectively used in subsequent construction activities, and combined with relevant standards to complete collaborative design work. In the design activities of the entire model, effectively promoting the sharing and interaction of digital information can ensure the frequency and number of exchanges between various software. We can effectively improve the production efficiency and quality of buildings and meet the common needs of construction units and owners. Only by strictly implementing the relevant technical standards, promoting the construction and development of collaborative work and implementing them effectively, can we truly promote the informatization construction of the construction industry, and provide a solid support for the construction and development of the construction industry in China.

\section{Specific Needs for Construction Drawings of Building Model Structures}

\subsection{Design Flow}

In the process of construction engineering, the construction of information model is extremely important. Compared with the traditional model design procedure, 
the design method of construction drawings has undergone significant changes on the original basis. In traditional drawing design methods, two-dimensional methods are generally used to complete this work. When the construction project is officially launched, it is also necessary to strictly follow a certain process to carry out the work. First of all, the designer should perform a refined analysis of the various data information in the drawings according to the actual situation, so as to better apply the computer programming model style [5]. Secondly, after completing the model design work, the staff should dynamically adjust the various data in the model according to the actual needs of the project. Third, we can determine the final construction structure drawing after obtaining accurate data and information. Two-dimensional model design work has played a vital role in the previous construction drawing design, but it requires more human resources and material resources, and requires more time for application. If some errors are prone to occur when the structural drawing is modified, the appearance of these uncertain factors will lead to different levels of problems in the subsequent work links.

When the new method of building information system model appears, construction engineering staff can have higher efficiency and quality when implementing drawing design work. In the process of continuous development of this new technology, compared with traditional working methods, this new method has many irreplaceable advantages. Firstly, the construction work itself has strong complexity, and the use of new technologies can ensure that the measured data information does not have errors. The construction of the information model will also be more complete. Once there is a situation that needs to be modified, the relevant data will also change according to the revised data, and the model drawing file can automatically update the content. Secondly, the construction of the information model is characterized by openness. Staff of various departments can watch the model structure under the permission of their authority, and can put forward some constructive opinions based on their observations. The sharing of information can make the connection between various departments closer.

\subsection{Description of Requirements}

In the process of building a building information model, the issues that staff need to consider are diversified. It should not only consider the way the physical structure model is constructed, but also the various related information, attribute values and management methods. The content contained in decoupled stock physical information is also more complicated, such as node information, axis network information, construction information, etc. [6]. In addition, in the related information, the company also needs to fully consider the attribute relationships and deemed relationships covered in the project. In the attribute information, the staff should also fully consider the quality of the materials used in the project and the load information. In the management of information, the owner information and user information need to be refined management. Only when these information are managed and analyzed in various aspects can the whole information model construction work be completed successfully, and the subsequent construction projects can be promoted to obtain higher quality. This is crucial to the future development of the entire project.

\section{Integrated Collaborative Design of Building Structure Based on Building Information Model}

\subsection{Design of Building Information Model}

The basic design of the building information model requires that the relevant design unit can fully conform to the existing standards in the industry and complete the design analysis of the logical structure. Simultaneously, in the actual model design, we need to fully enhance the attention and attention to operational efficiency and convenience. Through specific and complete design standards, effectively promote the construction and development of building information model design work. This method can greatly improve the authenticity and stability of the value information model, and it should also fully increase the attention to the structural construction design drawings when carrying out the logical structure design work. It mainly includes four important components: material information model, structural component, calculation result and attribute definition. The material information model mainly takes concrete, steel structure, etc. as the core. Structural components mainly include boards, columns, walls, doors and other components in the building structure. The calculation results mainly include the calculation results of the stress and the internal pressure that the current building needs to bear. The attribute definition mainly represents the attribute set, attribute definition and so on. The interrelation of the above four contents makes the basic form of architectural design more complete and concrete, and it also enables the building model to develop in a more complete and concrete direction. In addition, it can not only effectively promote the construction and development of the modern construction industry, but also provide a solid support for the stability of the construction industry.

\subsection{Construction Process of Building Information Model Platform}

In the process of carrying out architectural design work, the importance of the model design work carried out is quite huge. As an important design tool in the new era, building information model can be widely used in modern construction projects. We analyze the design process of the architectural plan given to the building information model platform. When carrying out the actual building model construction activities, the building information model is perfected and applied by the designer along with the building process. However, it can be seen from the basic situation that has been formed at this stage that it is an important transitional aid for the development of model 
construction for local specialties, local projects and local construction in the entire construction activity. As a result, in the follow-up specific design activities, it is necessary for companies to make scientific and reasonable management and control. Enterprises need to build corresponding building information models at different levels and time periods to essentially improve work quality and efficiency.

\subsubsection{Pre-concept Design Stage}

When carrying out conceptual design work, companies can present the content of the scheme involved with the help of building information models, which can be used to effectively improve work quality and efficiency, which has extremely important meaning and effect. While perfectly presenting the creative elements of architectural design activities, companies also need to fully increase their attention to building area, to the economic benefits of current construction projects, and to the trajectory of sunlight. This can be used to ensure that the building layout is accurate enough for subsequent specific implementation, and fundamentally improve the quality of construction projects.

\subsubsection{Scheme Design Stage}

At this stage, the building information model that enterprises need to apply plays a very important role in accurately analyzing the complexity of construction projects. It can help relevant workers analyze important content such as the feasibility of the plan and the contrast of the plan from a diversified perspective [7]. What'smore, it can accurately analyze and optimize the energy required for construction activities. This can ensure that construction companies can improve work quality and efficiency while taking on less economic pressure.

\subsubsection{Drawing Design Stage}

With the rapid development of modern society, the functions of construction projects are increasing day by day. Naturally, the complexity of buildings is gradually increasing in this situation, and the traditional twodimensional model can no longer effectively meet the actual development needs of modern society. Obviously, there are huge difficulties in the two-dimensional processing of complex graphics, which will also cause difficulties in subsequent observations, and seriously affect the basic quality of construction projects. The indepth application of building information models can greatly complete the design analysis of complex building structures, and ultimately effectively ensure the stability and accuracy of the design work.

\subsubsection{Professional Pipeline Integration}

Pipeline design can be said to be the core support to ensure the normal operation of construction activities. When working on the design and management of large-scale construction projects, the distribution of internal pipelines is usually not optimistic. The scientific application of building information model can solve this problem, and it can complete the precise design process of pipeline layout through its own three-dimensional model. This can ensure that it can develop in a more scientific and reasonable direction, and effectively improve the quality and efficiency of construction.

\section{Conclusion}

All in all, in the development process of the modern construction industry, the role of model design is quite huge, and it will be directly related to the quality of subsequent construction projects. How to effectively improve the stability of construction activities has become a key topic of modern society. Under this circumstance, the traditional architectural model is obviously unable to effectively meet the diverse needs of people. At the same time, the application of two-dimensional design schemes in increasingly complex projects is facing many difficulties. Various problems prove that construction companies must make a lot of optimization and innovation. It can be used to fundamentally improve the efficiency and quality of building structural design models. With the increasing level of productivity in modern society, the future of architecture is bound to develop in the direction of high-tech and informatization. Furthermore, related methods of architectural design will also be gradually improved, laying a solid support for the sustained and stable development of China's national economy.

\section{Acknowledgements}

General Project of Chongqing Higher Education Teaching Reform "Research and Practice of New Vocational Skills Training Model Based on School-Enterprise Cooperation" (No. 203195), Leading reseacher of the project group: Rulun Peng.

\section{References}

1. Zou Yafeng. Research on integrated collaborative design of building structure based on building information model[J]. Architecture, 2020(06): 73-74.

2. Zhang Haiyan. Discussion on the application of building information model in integrated collaborative design of building structure [J]. Building Technology, 2017, 48(08): 876-878.

3. Shen Hongfeng. Integrated collaborative design of building information model in building structure[J]. Sichuan Cement, 2017(07): 127.

4. Xia Xianyong. Application analysis of building information model in integrated collaborative design of building structure $[\mathrm{J}]$. Building Materials and Decoration, 2017(17): 76-77.

5. Liu Yang, Yan Sen. Research on the Cultivation Countermeasures of $1+\mathrm{X}$ Professional Grade Certificate for Construction Major-_ Take BIM Certificate of Building Information Model as an 
Example [J]. Journal of Liaoning Higher Vocational College, 2020, 22(12): 18-20+92 .

6. He Yan. Research on refined management of installation engineering cost based on building information model technology[J]. Ju She, 2020(33): 123-124.

7. Liu Mingqi, Wang Qiongfen. Preliminary study on the application of building information modeling technology in the construction phase of building engineering[J]. Building Science, 2020, 36(11): 159. 